\title{
Double-loop locking cross-stitch suture and suspension fixation for medial collateral ligament origin reconstruction
}

\author{
C.J. Li, B.C. Wang and D.Q. Liu \\ Department of Hand Surgery, Tangshan Second Hospital, Tangshan, \\ Hebei, China \\ Corresponding author: B.C. Wang \\ E-mail: baocangwang@126.com
}

Genet. Mol. Res. 13 (3): 5405-5412 (2014)

Received June 4, 2013

Accepted January 22, 2014

Published July 24, 2014

DOI http://dx.doi.org/10.4238/2014.July.24.20

\begin{abstract}
The purpose of this study was to summarize the clinical application and results of the double-loop locking cross-stitch suture and suspension fixation method for medial collateral ligament origin reconstruction. Thirty-six patients (21 males, 15 females) with an average age of 40 years (range $=17-58$ years), who underwent treatment for acute fracture of the medial collateral ligament at our hospital from February 2008 to May 2009, were included in this study. All patients presented unilateral injuries (17 right-sided, 19 left-sided) and underwent repair with the double-loop locking cross-stitch suture and suspension fixation method. All incisions in this group of patients healed by first intention. Thirty-two patients were followed up for 6-20 months (average $=12$ months). There were no reports of wound infection, ligament re-fracture or other complications in the follow-up period. Based on the Lysholm knee-scoring scale, the patients received a $100 \%$ excellent and good rating (20 patients - excellent score, 12 patients - good score) postoperatively. The advantages of the doubleloop locking cross-stitch suture and suspension fixation method are a smaller incision, reliable fixation, and early restoration of knee joint
\end{abstract}


stability. It is, therefore, an effective and low-risk method for the reconstruction of medial collateral ligament origin.

Key words: Medial collateral ligament; Ligament rupture; Loop locking; Knee joint

\section{INTRODUCTION}

The medial collateral ligament (MCL), which consists of an anterior longitudinal, a posterior superior oblique and a posterior inferior oblique band, is one of the most stable structures of the knee joint. The anterior longitudinal ligament, which arises from the adductor tubercle and below it and ends at the medial tibial, is covered by the pes anserinus tendon. It stretches mainly when the knee is extended to prevent genu valgum. The posterior superior oblique band starts from the femoral adductor tubercle and terminates in the inferior-posterior direction of the meniscus, at the joint capsule and the posterior margin of the tibial medial condyle. Some fibers of the posterior inferior oblique band are a continuation of the semimembranosus tendon, and converge at the end of the anterior longitudinal band. The posterior superior oblique and posterior inferior oblique bands are tensed when the angle of genuflexion is $30^{\circ}$, to prevent external rotation of the tibia. The MCL slides forward as the knee position changes from flexion to extension, and it slides backward as it changes from extension to flexion.

During knee flexion and extension, a partial fiber of the MCL remains tense at all times to keep the knee joint stable and to keep the femur and the tibia close, due to which the range of motion of the knee joint remains normal.

Lateral violence often leads to an MCL injury. Sports-induced MCL injury is the most common knee ligament injury requiring knee joint surgery (Marx et al., 2001). The American Medical Association Sports Medicine Committee has provided a classification for sports-induced MCL injury in the Manual of the Standard Nomenclature of Athletic Injuries. Based on this classification, an injury involving a small amount of torn ligament fibers with local tenderness and no instability is a first-degree injury. A second-degree injury involves more torn ligament fibers with evidence of instability and a third-degree injury is a complete laceration of the cruciate ligament with instability. The third-degree injury with obvious joint instability is further classified according to the degree of instability during the stress test. When the separation of the joint surfaces is $<5 \mathrm{~mm}$, it is referred to as $1+$ instability; when it is $5-10 \mathrm{~mm}$, it is referred to as $2+$ instability and when the separation of the joint surfaces is $>10 \mathrm{~mm}$, it is referred to as $3+$ instability. Fracture of the point of attachment on the femoral epicondyle accounts for a great proportion of the $3+$ instability cases. Currently, several treatments such as the use of gasket screw with a thorn, an anchoring nail, or a bio-resorbable interference screw are available. However, the need for special equipment limits their clinical use. Additionally, the transposition of the gracilis muscle and the semitendinosus tendon can cause significant damage. Thirty-six patients were treated for a simple, acute MCL fracture at our hospital from February 2008 to May 2009. These patients underwent repair with the double-loop locking cross-stitch suture and suspension fixation method, which did not cause damage to other tissues and strengthened the contact area between the ligament and the bone. 


\section{MATERIAL AND METHODS}

\section{Demographic data}

This study included 36 patients ( 21 males, 15 females) with an average age of 40 years (range $=17-58$ years) and presenting unilateral injuries (17 right-sided, 19 left-sided). The cause of injury included traffic accident (5 patients), pedestrian injury (11 patients), kicking injury ( 3 patients), crush injury ( 4 patients), and sprain injury (13 patients). All patients had a simple fracture of the attachment point on the femoral epicondyle. The knee valgus test was positive in all patients. In 31 cases, the site of the torn ligament was accurately identified with step-feeling. The torn ligament was located in the remaining 5 cases on performing MRI. The double loop locking cross-stitch suture and suspension fixation surgery was performed within $36 \mathrm{~h}$ to 8 days (average $=3.5$ days) after the injury.

\section{Surgical techniques}

\section{Anesthesia, surgical position and approaches}

The patients were operated in the lateral position under continuous epidural anesthesia or combined spinal-epidural anesthesia. The site of the disrupted ligament was identified through preoperative examination. A 7.0-cm longitudinal incision was made in the center of the torn ligament, all along its length until its attachment on the femoral medial condyle. A sharp dissection was performed to explore the torn end of the MCL.

\section{Drilling}

A Kirschner wire $(1.5 \mathrm{~mm})$ was used for drilling two holes obliquely at the proximal attachment point of the ligament. The two holes formed two bone tunnels that passed through the migrated site of the femoral shaft and the condyle (Figure 1).
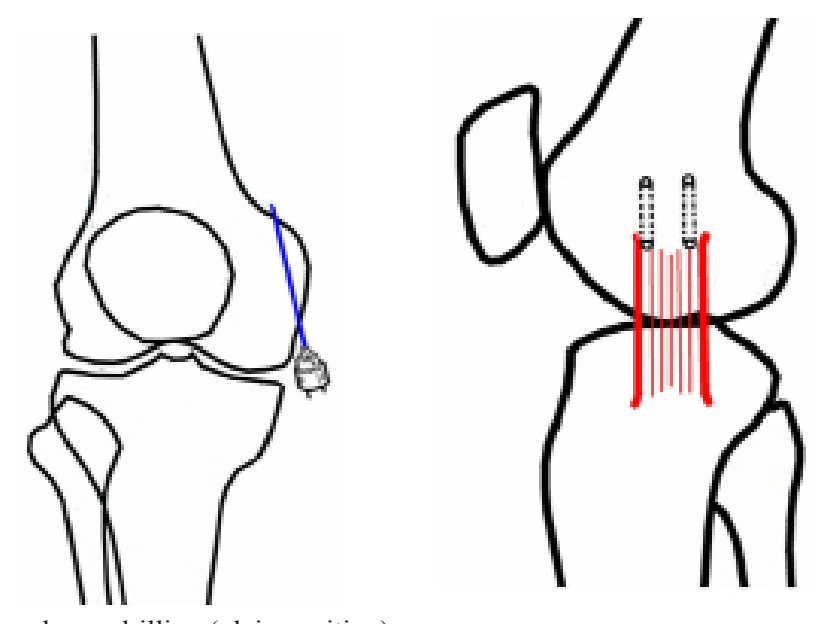

Figure 1. Surgical procedures: drilling (plain position). 


\section{Loop}

A double-loop was prepared by using tails made of $0 \#$ absorbable braided suture (Figures 2 and 3).

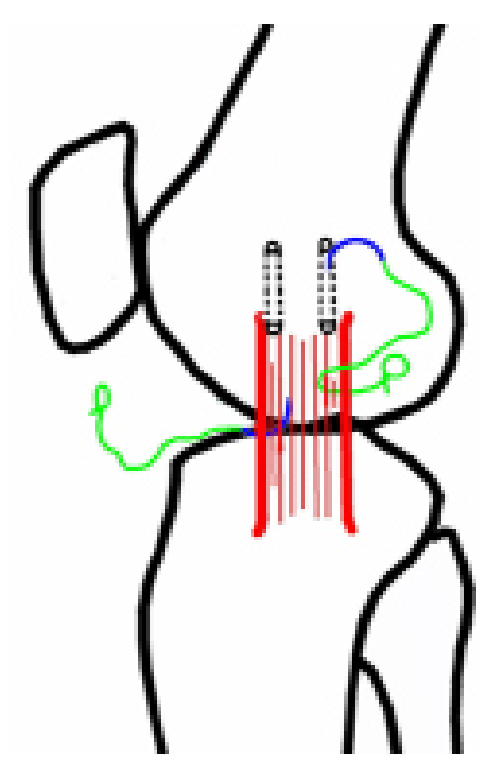

Figure 2. Surgical procedures: loop locking.
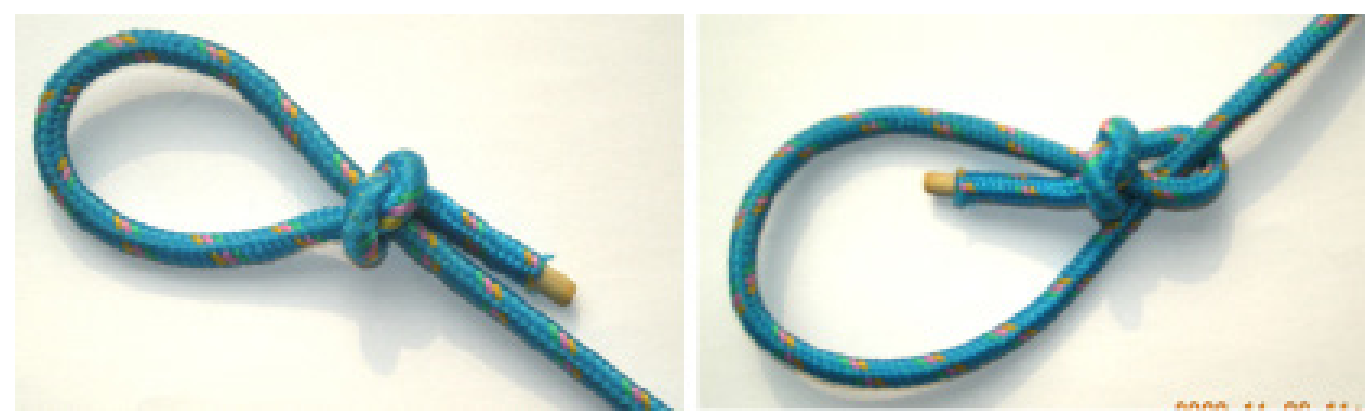

Figure 3. Surgical procedures: the suture is passed through the loop to lock partial medial collateral ligament.

\section{Locking}

The MCL was sutured transversally with an absorbable suture with loops in the caudo-medial part (1.5-2 cm from the injury site). The absorbable suture was then passed through the loop to lock the MCL partially. The needle was pulled out at the torn end of the ligament, and a tail suture of about $10.0 \mathrm{~cm}$ was reserved in the torn end at the same time. The abovementioned procedure was repeated at different levels of the ligament (Figures 4 and 5). 


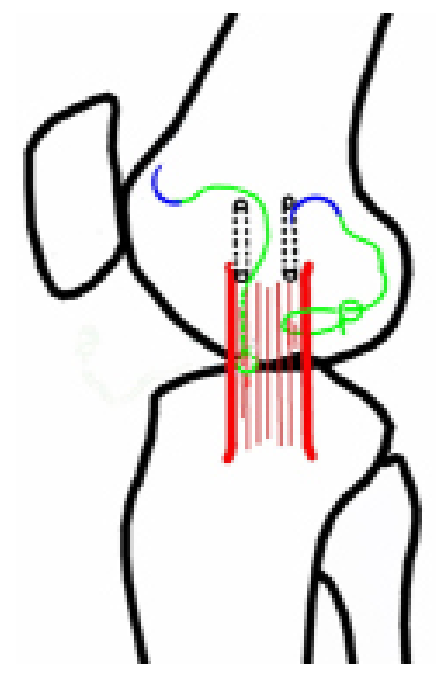

Figure 4. Surgical procedures: the absorbable sutures are passed through the bone tunnels by using a crude syringe needle and a lumbar puncture needle.

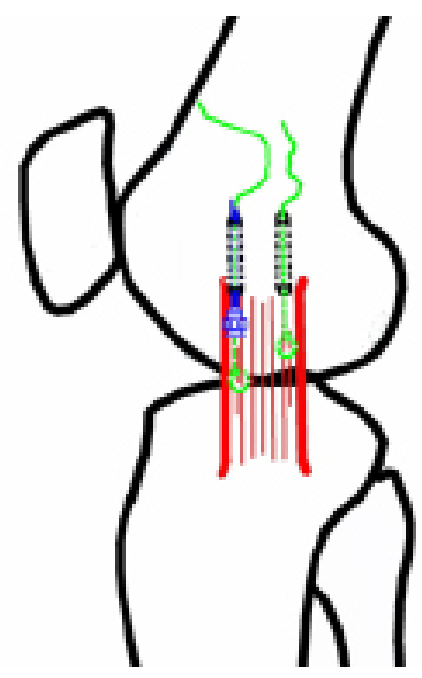

Figure 5. Surgical procedures: two absorbable sutures are passed through bone tunnels and tied tightly.

\section{Suspension fixation}

A syringe needle was used to pass the two standby tails of the suture through the two bone tunnels, which had been drilled at the migrated site of the femoral shaft and the condyle (Figure 3). A tight knot was then tied, maintaining the functional position of the knee joint (Figure 6). The remaining parts of the torn ligament were repaired with an 8-shaped suture. The knee valgus test was conducted under direct vision to inspect the degree of firmness of the 
MCL. The incision was closed after being washed and pressure bandaged with sterile material. The injured limb was then supported in a plaster.

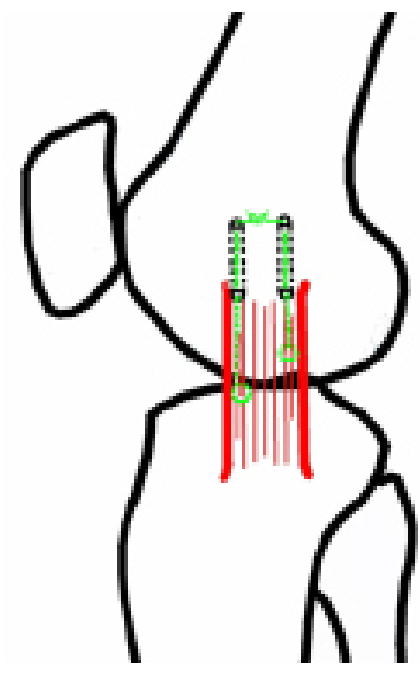

Figure 6. Models of loop locking.

\section{Postoperative treatment}

A long plaster splint was applied postoperatively to the operated limb for fixation with the genuflex angle set at 15-20 degrees. The patients were prescribed routine anti-inflammatory medication and early active quadriceps exercises simultaneously. Knee flexion and extension activities were performed by the patient after plaster removal at 4 weeks.

\section{RESULTS}

All incisions in this study healed by first intention. Thirty-two cases were followed up for 6-20 months (average $=12$ months). There were no reports of wound infection, ligament re-fracture or other complications in the follow-up period, and the stress test at $30^{\circ}$ knee valgus flexion was negative in all patients. The average joint space of both medial knee joints reduced to $5.6 \mathrm{~mm}$ on radiographs compared to the preoperative joint space. On an average, the score of the 32 selected cases on the Lysholm knee-scoring scale (Marx et al., 2001), during the postoperative evaluation was higher than 83 points with a $100 \%$ excellent and good rating (excellent score - 20 patients, good score - 12 patients). None of the 32 patients had a fair or poor score (Table 1).

Table 1. Average postoperative scores of the cases selected.

\begin{tabular}{lccc}
\hline $\begin{array}{c}\text { Valgus stress test with knee } \\
\text { in flexion (negative/positive) }\end{array}$ & $\begin{array}{c}\text { Reduction of the joint space } \\
\text { of both medial knee joint under } \\
\text { stress on radiographs (mm) }\end{array}$ & $\begin{array}{c}\text { Scores on the Lysholm } \\
\text { knee-scoring scale (points) }\end{array}$ \\
\hline Result & Negative & $<5.6$ & $>83$ \\
\hline
\end{tabular}




\section{DISCUSSION}

\section{MCL injury}

MCL injury is the most common knee ligament injury. It usually occurs in case of lateral trauma to the knee abduction of the leg with the knee in minimal flexion. MCL is extremely important for knee stability. The repair of an MCL fracture is crucial to maintaining knee stability and functional recovery (Crowe et al., 1997). A large number of clinical reports showed that the most common site of injury of the MCL was near its femoral attachment point (Roth et al., 2000; Fu et al., 2001; Sukoff, 2001; Li et al., 2003). It was reported that early surgical repair was an effective method for treatment of MCL injury of knee joint (Parker and Gurusamy, 2005). Conventional treatments included anchoring nail or block button fixation (Al-Munajjed et al., 2008; Simmermacher et al., 2008; Zou et al., 2010), arthroscopic treatment with a tight suture (Casaletto and Gatt, 2004), transposition of gracilis and semitendinosus tendon (Galibert et al., 1987; Hashimoto et al., 1988; Boldin et al., 2003; Ha et al., 2006; Saarenpǎă et al., 2007; Eser et al., 2010; Wang et al., 2010). However, the need for special equipment or materials and compensatory tissues limits their clinical use.

\section{Double-loop locking cross-stitch suture and suspension fixation for medial collateral ligament reconstruction}

\section{Indication: avulsion fracture of the attachment point of the MCL on the medial femoral condyle}

Advantages: 1) The double-loop locking technique utilizes a locking suture, which locks and pre-tenses some ligament fibers. This method has strong anti-slip and anti-crack effects that increase the tensile strength of the repaired ligaments and prevent gap formation leading to early functional restoration of the knee joint. 2) Loop locking locks less tendon fibers and two locking cross-stitch sutures are performed at different levels, which leads to less impairment of ligament blood supply, and thereby aids ligament recovery. 3) Based on the anatomical characteristics of the medial femoral condyle (Cierny III, 1990; McNally et al., 1993; Schimmel et al., 1998; Sporer et al., 2006), bone tunnels are created in the femoral condyle and the suture material is passed through them for fixation with the suspending method. This method is reliable and has a low risk of slippage or movement. 4) The technique does not require steel wires, bone anchors or other special materials and equipment. Therefore, there is no risk of development of foreign body complications and related secondary surgeries. It also reduces chances of developing a wound infection, thereby reducing therapeutic costs and improving patient satisfaction. 5) The surgery does not damage the gracilis, the semitendinosus, or the peripheral tissues. 6) The surgery does not need special materials, only absorbable sutures, and the operation is simple and easy even for novice surgeons.

Key points: The drilling angle of the bone tunnels needs specific attention because if the angle is too large, the exit of the tunnel is far from the entrance, making threading and knotting more difficult. If the angle is too small, the chances of bone splitting increase. Because the torn end of the ligament is irregular, the location of each locking suture should be accurate, to ensure that the loops are locked at different levels rather than at the same level, 
which can limit the blood supply of the ligament and its recovery. The position of the injured limb is fixed when the tail suture is tied.

Shortcomings: Although this surgical procedure is an effective method for repairing an injured tendon or ligament, further studies are needed to investigate the biomechanical characteristics of loop locking.

\section{REFERENCES}

Al-Munajjed AA, Hammer J, Mayr E, Nerlich M, et al. (2008). Biomechanical characterisation of osteosyntheses for proximal femur fractures: helical blade versus screw. Stud. Health Technol. Inform. 133: 1-10.

Boldin C, Seibert FJ, Fankhauser F, Peicha G, et al. (2003). The proximal femoral nail (PFN) - a minimal invasive treatment of unstable proximal femoral fractures: a prospective study of 55 patients with a follow-up of 15 months. Acta Orthop. Scand. 74: 53-58.

Casaletto JA and Gatt R (2004). Post-operative mortality related to waiting time for hip fracture surgery. Injury 35: 114120.

Cierny G III (1990). Chronic osteomyelitis: results of treatment. Instr. Course Lect. 39: 495-508.

Crowe MJ, Bresnahan JC, Shuman SL, Masters JN, et al. (1997). Apoptosis and delayed degeneration after spinal cord injury in rats and monkeys. Nat. Med. 3: 73-76.

Eser O, Aslan A, Cosar M, Kalkan E, et al. (2010). Kyphoplasty method for the treatment of traumatic osteoporotic vertebral compression fractures: case report. Ulus. Travma. Acil. Cerrahi. Derg. 16: 95-97.

Fu Q, Hou TS, Lu KW, He SS, et al. (2001). Apoptosis of neural cells after acute spinal cord injury in rats. Chin. J. Traumatol. 17: 222-224.

Galibert P, Deramond H, Rosat P and Le Gars D (1987). Preliminary note on the treatment of vertebral angioma by percutaneous acrylic vertebroplasty. Neurochirurgie 33: 166-168.

Ha KY, Lee JS, Kim KW and Chon JS (2006). Percutaneous vertebroplasty for vertebral compression fractures with and without intravertebral clefts. J. Bone Joint Surg. Br. 88: 629-633.

Hashimoto T, Kaneda K and Abumi K (1988). Relationship between traumatic spinal canal stenosis and neurologic deficits in thoracolumbar burst fractures. Spine 13: 1268-1272.

Li CC, Sun XB, Hang RC, Lian QG, et al. (2003). Effects of hyperbaric oxygen pretreatment on cytokines production in neonatal rat microglias in vitro. Acad. J. Sec. Mil. Med. Univ. 24: 58-60.

Marx RG, Jones EC, Allen AA, Altchek DW, et al. (2001). Reliability, validity, and responsiveness of four knee outcome scales for athletic patients. J. Bone Joint Surg. Am. 83-A: 1459-1469.

McNally MA, Small JO, Tofighi HG and Mollan RA (1993). Two-stage management of chronic osteomyelitis of the long bones. The Belfast technique. J. Bone Joint Surg. Br. 75: 375-380.

Parker MJ and Gurusamy K (2005). Modern methods of treating hip fractures. Disabil. Rehabil. 27: 1045-1051.

Roth KA, Kuan C, Haydar TF, D'Sa-Eipper C, et al. (2000). Epistatic and independent functions of caspase-3 and Bcl$\mathrm{X}(\mathrm{L})$ in developmental programmed cell death. Proc. Natl. Acad. Sci. U. S. A. 97: 466-471.

Saarenpăă I, Heikkinen T and Jalovaara P (2007). Treatment of subtrochanteric fractures. A comparison of the Gamma nail and the dynamic hip screw: short-term outcome in 58 patients. Int. Orthop. 31: 65-70.

Schimmel JW, Buma P, Versleyen D, Huiskes R, et al. (1998). Acetabular reconstruction with impacted morselized cancellous allografts in cemented hip arthroplasty: a histological and biomechanical study on the goat. J. Arthroplasty 13: 438-448.

Simmermacher RK, Ljungqvist J, Bail H, Hockertz T, et al. (2008). The new proximal femoral nail antirotation (PFNA) in daily practice: results of a multicentre clinical study. Injury 39: 932-939.

Sporer SM, O'Rourke M, Chong P and Paprosky WG (2006). The use of structural distal femoral allografts for acetabular reconstruction. Surgical technique. J. Bone Joint Surg. Am. 88 (Suppl 1; Pt 1): 92-99.

Sukoff MH (2001). Effects of hyperbaric oxygenation. J. Neurosurg. 95: 544-546.

Wang G, Yang H and Chen K (2010). Osteoporotic vertebral compression fractures with an intravertebral cleft treated by percutaneous balloon kyphoplasty. J. Bone Joint Surg. Br. 92: 1553-1557.

Zou J, Mei X, Gan M, Wang G, et al. (2010). Is kyphoplasty reliable for osteoporotic vertebral compression fracture with vertebral wall deficiency? Injury 41: 360-364. 